\title{
Efecto de Remedios Homeopáticos y Gentamicina en Diarreas en Crías Porcinas
}

\author{
Effect of Homeopathic Remedies and Gentamicin in Diarrhea in Piglets \\ Florangel Vidal F. ${ }^{1,2}$, Iván Peña G. ${ }^{1}$, Aliesky Hernández R. ${ }^{1}$, José Bertot V. ${ }^{1}$, \\ Daylitt Noda S.V. ${ }^{1}$
}

\section{Resumen}

El estudio tuvo como objetivo evaluar el efecto de nux vomica y el complejo homeopático TratHo ${ }^{\circledR}$ cólera, en comparación con la gentamicina al 5\% en la diarrea de lechones. Se trabajó con 150 lechones de cinco días de nacidos que presentaron diarrea en una unidad porcina de Camagüey, Cuba. Los lechones fueron distribuidos en tres grupos, cada uno de 50 individuos. A los animales del grupo I se le administró nux vomica a la $30 \mathrm{CH}$ en dosis de 5 gotas sublinguales cada $12 \mathrm{~h}$ el $1^{\text {er }}$ día y luego cada $24 \mathrm{~h}$ hasta el $5^{\circ}$ día. En el grupo II se les administró TratHo® colera a la $30 \mathrm{CH}$ en dosis de 5 gotas sublinguales cada $12 \mathrm{~h}$ el $1^{\text {er }}$ día y luego cada $24 \mathrm{~h}$ hasta el $5^{\circ}$ día. En el grupo III se les administró Gentamicina al 5\% por vía intramuscular, en dosis de 2 a $4 \mathrm{mg} / \mathrm{kg}$ de peso vivo, cada $12 \mathrm{~h}$ durante 5 días. En todos los casos, los lechones fueron rehidratados con solución Ringer, vía intraperitoneal, en dosis de 100 a 300 ml, según el grado de deshidratación. Se evaluó la evolución clínica, el apetito y la deshidratación sin llegar a encontrarse diferencias estadísticas entre grupos; no obstante, la terapia homeopática resultó efectiva y más económica que el tratamiento con antibiótico.

Palabras clave: diarrea, nux vomica, TratHo® colera, homeopatía

\section{Abstract}

The study aimed to evaluate the effect of nux vomica, the homeopathic complex TratHo ${ }^{\circledR}$ cholera and Gentamicin 5\% in the treatment of diarrhea in piglets. A total of 150 piglets of 5 days old that showed diarrhea in a pig unit in Camagüey, Cuba, were evaluated. The animals were distributed in three groups of 50 each. In group I, piglets were treated

${ }^{1}$ Facultad de Ciencias Agropecuarias, Universidad de Camagüey «Ignacio Agramonte Loynaz», Camagüey, Cuba

${ }^{2}$ E-mail: florangel.vidal@reduc.edu.cu

Recibido: 23 de diciembre de 2015

Aceptado para publicación: 20 de julio de 2016 
with nux vomica $30 \mathrm{CH}$ in a dose of 5 drops sublingual every $12 \mathrm{~h}$ the $1^{\text {st }}$ day and then every $24 \mathrm{~h}$ until the $5^{\text {th }}$ day. In group II, piglets were treated with to TratHo ${ }^{\circledR}$ cholera 30 $\mathrm{CH}$ in a dose of 5 drops sublingual every $12 \mathrm{~h}$ the $1^{\text {st }}$ day and then every $24 \mathrm{~h}$ until the $5^{\text {th }}$ day. In group III, piglets were treated with gentamicin 5\% intramuscularly, 2 to $4 \mathrm{mg} / \mathrm{kg}$ every $12 \mathrm{~h}$ for 5 days. In all cases, piglets were rehydrated with lactated Ringer's solution intraperitoneally, using 100 to $300 \mathrm{ml}$ depending of the degree of dehydration. The clinical evolution, appetite and dehydration were analyzed without observing significant differences between groups. However, the homeopathic therapy was effective and cheaper than the antibiotic treatment.

Key words: diarrhea, nux vomica, TratHo cholera, homeopathy

\section{INTRODUCCIÓN}

En general, uno de los grandes problemas que enfrenta la producción porcina es la alta prevalencia de las enfermedades que afectan a los lechones en su primera etapa de vida, como consecuencia de deficiencias en la aplicación de los programas de control previstos. Es reconocido que los animales pasan por un conjunto de cambios importantes entre el nacimiento y el destete y estos deben responder adecuadamente para restablecer el equilibrio fisiológico interno (homeostasis) (Del Francia, 1988; Montfort Cabello, 2014). La ocurrencia de enfermedades se revierte en pérdidas económicas considerables por incremento en la tasa de mortalidad, retardo en el crecimiento, pobre conversión alimenticia y, adicionalmente, por los costos en medicación (Rodríguez Pereira, 2015).

Las enfermedades entéricas son un problema común en todas las etapas de la producción porcina moderna en todo el mundo. La diarrea es una consecuencia de la disfunción en el transporte de agua y electrólitos a nivel del intestino (Ruiz Camejo, 2015). En la producción porcina cubana, la principal causa de muerte de lechones la constituye las enfermedades gastrointestinales, de allí que se trazan estrategias para su prevención partiendo de un diagnóstico eficaz (Pluske et al., 2006).
Una de las enfermedades gastrointestinales más frecuentes en el cerdo es la colibacilosis (Barreto, 2007; Valdés et al, 2012). En este sentido, Blood et al. (1988) y Gallego et al. (2009) definen como colibacilosis enterotóxica a las diarreas producidas por Escherichia coli en lechones. Para su control se emplean medidas profilácticas y terapéuticas, incluyendo el uso de sulfas e hidratación con terapia líquida y antimicrobiana (Burgard, 1987; Ancízar, 2010; Ancízar et al., 2012).

Los antibióticos desempeñan una importante función en la reducción de la mortalidad e incidencia de enfermedades infecciosas; sin embargo, su uso indiscriminado y la falta de conocimiento sobre una adecuada administración puede provocar la presencia de residuos tóxicos en la carne de los animales beneficiados, ocasionando un riesgo para la salud de los consumidores por el desarrollo de resistencia de los microorganismos (Barreto y Rodríguez, 2007; León, 2014).

López et al. (2014) señalan que en Cuba se han valorado diferentes productos homeopáticos como alternativas profilácticas, cuyos resultados conducen a la extensión del empleo de bioterápicos. Los remedios homeopáticos se emplean en muchos países como terapia eficaz, económica y libre de efectos secundarios importantes (Legoni, 1985; Manhosa et al., 2014; Vidal et al., 2015). Atendiendo a lo antes expuesto, el pre- 
sente trabajo tuvo como objetivo evaluar el efecto de Nux vomica, TratHo ${ }^{\circledR}$ colera en comparación con gentamicina en las diarreas de crías porcinas.

\section{Materiales y Métodos}

El estudio se realizó en la unidad Rescate de Sanguily, municipio Camagüey, Cuba, entre enero de 2013 y mayo de 2015. Se efectuó un ensayo clínico fase II abierto, no secuencial, aleatorizado, a partir de una muestra de 150 lechones, de ambos sexos y de cinco días de nacidos. Todos los lechones seleccionados consumieron el calostro adecuadamente, presentaban diarrea y permanecieron con sus madres hasta el destete, sin suspender lactación.

La unidad porcina tenía 500 reproductoras. El agua de bebida era a través de tetinas. La limpieza de los pisos se realizaba dos veces al día, en la mañana se limpia y se pasa cal viva $(\mathrm{CaO})$ y en la tarde se limpia con agua a presión. La cama de los lechones era cambiada diariamente, manteniendo una buena higiene.

En los casos de diarreas, usualmente enfermaban seis o siete lechones como promedio por marrana. Estos lechones fueron distribuidos en forma aleatoria a cada uno de los tratamientos. Las madres no presentaron diarrea en ninguno de los casos.

Para determinar la presencia de patógenos causantes del síndrome diarreico, se tomaron muestras de material fecal en las crías diarreicas y en el laboratorio se hicieron los análisis microbiológicos. Sin embargo, no se hizo antibiograma de las cepas prevalentes, utilizándose la gentamicina como antibiótico para el tratamiento de las diarreas, antibiótico recomendado en Cuba para el tratamiento de la colibacilosis.

La población experimental fue dividida en tres grupos:
- Grupo 1: 50 lechones que recibieron el tratamiento de nux vomica a las $30 \mathrm{CH}$, a razón de 5 gotas sublinguales cada $12 \mathrm{~h}$ el primer día y luego cada $24 \mathrm{~h}$ hasta el quinto día (Cuesta et al., 2007).

- Grupo 2: 50 lechones que recibieron el tratamiento con el complejo homeopático (TratHo ${ }^{\circledR}$ cólera) a la $30 \mathrm{CH}$, a razón de 5 gotas sublinguales cada $12 \mathrm{~h}$ el primer día y luego cada $24 \mathrm{~h}$ hasta el quinto día.

- Grupo 3: 50 lechones tratados con gentamicina al $5 \%$, vía I.M., en dosis de 2 a $4 \mathrm{mg} / \mathrm{kg}$ de peso vivo, cada $12 \mathrm{~h} \mathrm{du}-$ rante 5 días.

En todos los casos, los animales fueron hidratados con Solución Ringer Lactato por vía intraperitoneal, en volumen de acuerdo al grado de deshidratación.

Se evaluó el efecto terapéutico según la proporción de animales recuperados al tercero y quinto día de iniciado el tratamiento, clasificándose el estado clínico en asintomático, mejorado y empeorado o fallecido. Se consideró que el tratamiento fue efectivo si el lechón estuvo asintomático al quinto día del tratamiento. Además, se evaluó el comportamiento del apetito y la deshidratación.

En los estados clínicos se consideró lo siguiente:

- Asintomático: ausencia de diarrea, no presencia de deshidratación, respuesta a los estímulos de ligera intensidad, no evidencia de dolor abdominal.

- Mejorado: disminución de la diarrea en frecuencia y consistencia, desaparición de la deshidratación, respuesta a los estímulos de ligera intensidad, ausencia de dolor abdominal.

- Empeorado o fallecido: aumento en la intensidad de la diarrea, deshidratación moderada a intensa, marcada depresión, dolor abdominal, escalofríos, síndrome de estupor. 
Se calcularon los gastos, analizándose el costo-beneficio del tratamiento. Se consideró la duración del tratamiento en días y los costos por día y por animal, así como por el total del tratamiento, según Trujillo et al. (2007).

Para la determinación del costo del tratamiento con las terapias homeopáticas, se tomó en consideración que el frasco de 120 $\mathrm{ml}$ tenía un valor de $\$ 0.75$ (pesos cubanos), y que $1 \mathrm{ml}$ equivalía a 16 gotas. Para el tratamiento con gentamicina, el costo de $1 \mathrm{ml}$ fue de $\$ 0.02$ y se requería $0.26 \mathrm{ml}$ por lechón/día. Asimismo, el valor del cerdo en categoría de cría era $\$ 6.25$, el salario mensual del técnico veterinario era $\$ 260.00(\$ 0.02 / \mathrm{min})$ y se requería 1.5 min para tratar un lechón.

Los datos fueron analizados con la prueba de Chi cuadrado, con un nivel de significación de $\mathrm{p}<0.05$ (SPSS, 2006).

\section{Resultados y Discusión}

En la detección del proceso diarreico, los lechones presentaban piloerección, pérdida de la vitalidad, apetito disminuido, ligera deshidratación y temperatura normal o ligeramente aumentada. Las heces se caracterizaron por ser en su mayoría líquidas, predominando el color amarillo-carmelitoso con ligera o moderada presencia de mucus. Estos síntomas coinciden con los descritos por Cabrera y García (2010) para la colibacilosis. Las muestras de heces resultaron positivas a Escherichia coli, confirmando el diagnóstico presuntivo realizado al comenzar el experimento.

El Cuadro 1 presenta la distribución porcentual de la evolución del proceso diarreico de los lechones según el tratamiento empleado, sin que se haya encontrado diferencias significativas entre tratamientos; de allí que los resultados demuestran una similar efectividad con el uso de Nux vómica y de TratHo ${ }^{\circledR}$ cólera, en comparación con el uso de antibióticos en el tratamiento de la diarrea del lechón.

Nux vomica y el complejo homeopático (Tratho ${ }^{\circledR}$ cólera) son remedios homeopáticos de probada acción sobre casos con trastornos digestivos. Se les emplea en casos con sintomatología de diferentes tipos de diarreas, trastornos de la digestión de las grasas, anorexia, dolor abdominal, gastritis, dispepsias agudas, infección por bacterias entéricas patógenas y colibacilosis (Linares y Briones, 2004).

La mayoría de las muertes se produjo por recrudecimiento de los síntomas, de allí la importancia de extremar los cuidados, así como determinar las causas que puedan originarlas (Ruiz Camejo, 2015).

La efectividad de los medicamentos homeopáticos en el tratamiento del síndrome diarreico en el cerdo encontrado en el presente estudio es coincidente con otros estudios (Vivanco, 2002; Vidal et al., 2005, 2014; Pérez et al., 2006). La homeopatía en Cuba tiene una larga historia de tratamiento en animales con diarreas, reportándose el uso de Arsenicum album a la $6 \mathrm{CH}$ (Pérez et al., 2006), así como de otros medicamentos, alcanzado recuperaciones de 73 a $95 \%$ de los lechones afectados (Vidal et al., 2005; Palacio et al., 2012; Peña et al., 2012).

El Cuadro 2 muestra la distribución porcentual del apetito al tercer y quinto día del tratamiento según el tipo de medicamento empleado. El apetito se encontraba disminuido al inicio del estudio, pero al $5^{\circ}$ día se llegó a recuperar en el $94-98 \%$ de los animales. No se observó diferencia estadística entre tratamientos. Diversos estudios cubanos corroboran que animales con trastornos digestivos recuperan el apetito al ser tratados con medicamentos homeopáticos, por cuanto actúan sobre el organismo estimulando las reacciones defensivas del sistema inmunitario y de regulación nerviosa (Vidal et al., 2004; Gutiérrez et al., 2007; Peña et al., 2012; López, 2014; Morales, 2014). 
Cuadro 1. Distribución porcentual (\%) de los animales de acuerdo a la medicación contra diarrea según la evolución clínica al $3^{\text {er }}$ y $5^{\circ}$ día de tratamiento $(n=50$ por grupo)

\begin{tabular}{lccccc}
\hline \multirow{2}{*}{ Tratamiento $^{1}$} & \multicolumn{3}{c}{ Tercer día } & \multicolumn{2}{c}{ Quinto día } \\
\cline { 2 - 6 } & Asintomático & Mejorado & Empeorado & Asintomático & Muerto \\
\hline Nux vomica & 90 & 6 & 4 & 96 & 4 \\
TratHo cólera & 96 & 2 & 2 & 98 & 2 \\
Gentamicina 5\% & 88 & 6 & 6 & 94 & 6 \\
\hline
\end{tabular}

${ }^{1}$ No hubo diferencia estadística entre tratamientos

Cuadro 2. Distribución porcentual (\%) de lechones de acuerdo a la medicación contra diarrea según apetito al $3^{\text {er }}$ y $5^{\circ}$ día de tratamiento ( $\mathrm{n}=50$ por grupo)

\begin{tabular}{lcccc}
\hline Tratamiento $^{1}$ & \multicolumn{2}{c}{ Tercer día } & \multicolumn{2}{c}{ Quinto día } \\
\cline { 2 - 5 } & Con apetito & Sin apetito & Con apetito & Sin apetito \\
\hline Nux vomica & 90 & 10 & 96 & 4 \\
TratHo cólera & 96 & 4 & 98 & 2 \\
Gentamicina 5\% & 88 & 12 & 94 & 6 \\
\hline
\end{tabular}

${ }^{1}$ No hubo diferencia estadística entre tratamientos

Cuadro 3. Análisis económico de la medicación contra diarrea en lechones según el tipo de tratamiento

\begin{tabular}{lccc}
\hline Tratamiento & Gastos totales & $\begin{array}{c}\text { Costo por } \\
\text { lechón }\end{array}$ & $\begin{array}{c}\text { Gastos en } \\
\text { medicamentos }\end{array}$ \\
\hline Nux vomica & $\$ 99.65$ & $\$ 1.99$ & $\$ 0.65$ \\
TratHo cólera & $\$ 93.65$ & $\$ 1.87$ & $\$ 0.65$ \\
Gentamicina 5\% & $\$ 105.76$ & $\$ 2.11$ & $\$ 2.40$ \\
\hline
\end{tabular}

Entre el 88 y $96 \%$ de los lechones presentaban una deshidratación ligera en el primer día del tratamiento, observándose entre 2 y $6 \%$ de deshidrataciones severas al quinto día del tratamiento, sin diferencia estadística entre grupos. Los lechones con deshidrata- ción severa llegaron a morir. La deshidratación ocurre debido a la carencia de líquidos corporales y electrolitos (sodio, cloruro y potasio), necesarios para que el organismo cumpla con sus funciones normales (Cuesta et al., 2007). 
El Cuadro 3 presenta el resultado del análisis económico según los criterios establecidos por Trujillo et al. (2007). El tratamiento más económico y eficaz fue con el complejo homeopático a la $30 \mathrm{CH}$. El TratHo ${ }^{\circledR}$ cólera no causa efectos colaterales adversos en los animales y el costo-beneficio observado en el presente estudio es respaldado por otros trabajos (Vidal et al., 2004; Peña et al., 2012; Morales, 2014; López, 2014) al aplicar tratamientos homeopáticos para combatir el síndrome diarreico en crías porcinas.

\section{Conclusiones}

- La Nux vomica y el complejo homeopático (Tratoho® cólera) mostraron una efectividad similar a la gentamicina en el tratamiento de las diarreas y en el mejoramiento del estado general de salud de los lechones.

- Los tratamientos homeopáticos resultaron más económicos que el tratamiento convencional.

\section{Literatura Citada}

1. Ancízar J, Rodríguez $N$, Valdés D, Nuevas M, Prieto F. 2012. Principales enfermedades diagnosticadas en los cerdos de los sectores privado y cooperativo en las provincias habaneras durante los años 2010-2011. En: V Seminario Internacional Porcinocultura Tropical. Cuba.

2. Ancízar J. 2010. Las enterotoxemias por Escherichia coli en el cerdo. Una patología que aunque bien conocida causa grandes pérdidas a la ganadería porcina. Casos diagnosticados en el CENEDI durante los años 2008 y 2009. En: IV Seminario Internacional Porcinocultura Tropical. Cuba.

3. Barreto G. 2007. Escherichia coli, últimos 122 años. Rev Prod Anim 19: 55-57.
4. Barreto G, Rodríguez H. 2007. La cápsula bacteriana, algo más que una estructura no esencial. Rev Prod Anim 20: 69-80.

5. Blood DC, Radostitis OM, Henderson JA, Arundel JH, Gay CC. 1988. Medicina veterinaria. $6^{\mathrm{a}}$ ed. México DF: Nueva Ed Interamericana. 1413 p.

6. Burgard H. 1987. Digestive disorders of alimentary and infectious aetiologi and their treatment by the homeopathic veterinarian in small animal practice. Biol Tiermedizin 4: 2-13.

7. Cabrera Y, García A. 2010. Incidencia de los trastornos gastrointestinales en la masa porcina cubana.En: IV Seminario Internacional Porcinocultura Tropical. La Habana, Cuba.

8. Cuesta M, Montejo E, Bubergel J. 2007. Medicina Interna Veterinaria II. Cap 12. Homeopatía en Medicina Veterinaria. La Habana, Cuba: Félix Barela. p 289-306.

9. Del Francia F. 1988. Homeopathie et medicine veterinaire. J Org Med Homeopathique Int 3: 21-25.

10. Gallego C, Martínez M, Castro M. 2009. Empleo del Ligmed-A en el control de diarreas porcinas. Bol Téc Porcino 11: 6 .

11. Gutiérrez, R, Vidal F, Peña I. 2007. Eficacia de la China officinalis a la 30 $\mathrm{CH}$ en el tratamiento de la disentería porcina en preceba. En: VI Congreso Internacional de Ciencias Veterinarias. La Habana, Cuba.

12. Hampson D, Hopwood I, Pluske E. 2006. Relación entre la microbiótica intestinal, el pienso y la incedencia de diarreas, y su influencia sobre la salud del lechón tras el destete. Rev Porcicultura 23: 4-21.

13. Legoni N. 1985. Effect and efficacy of homeopathic drugs in veterinary medicine. Parkt Tierarzt 66: 541-548.

14. León C. 2014. Medicina naturalista y bioenergética. En: XXIV Congreso Paramericano de Ciencias Veterinarias. La Habana, Cuba. 
15. Linares F, Briones F. 2004. Vademécum de productos homeopáticos. Cienfuegos, Cuba: Labiofam. 6 p.

16. López R, Morales M, Vidal F. 2014. La homeopatía en la producción animal. En: XXIV Congreso Panamericano de Ciencias Veterinarias. La Habana, Cuba.

17. Manhosa F, Domínguez C, Galvani $A$. 2014. Enseñanza de la homeopatía en Brasil. En: XXIV Congreso Panamericano de Ciencias Veterinarias. La Habana, Cuba.

18. Montfort Cabello H. 2014. El camino de la salud a la enfermedad. La homeopatía de México 83(690): 5-13.

19. Morales M. 2014. Uso de la homeopatía en animales de producción En: XXIV Congreso Panamericano de Ciencias Veterinarias. La Habana, Cuba.

20. Palacio D, Vidal F, Peña I, Vázquez A, Bertot J, González C. 2012. Efectividad del Arsenicum albula al $6 \mathrm{CH}$ en el tratamiento de las crías porcinas. En: V Seminario Internacional de Porcinocultura Tropical. La Habana, Cuba.

21. Peña I, Vidal F, Henández A, de la Torre R. 2012. Efectividad de la terápia homeopática en los trastornos digestivos en el cerdo criollo. En: V Seminario Internacional de Porcinocultura Tropical. La Habana, Cuba.

22. Rodríguez Pereira P. 2015. Eficacia de tres antihelmínticos frente a Macracanthorhynchus hirudinaceus en cerdos de crianza privada. En: VI Seminario Internacional de Porcicultura Tropical. La Habana, Cuba.

23. Ruiz Camejo R. 2015. Comportamiento de los parásitos gastrointestinales del cerdo en la crianza de traspatio. En: VI Seminario Internacional de Porcicultura Tropical. La Habana, Cuba.

24. SPSS. 2006. SPSS for Windows. [Internet] Disponible en: http:// www.spss.com

25. Trujillo C, Cuesta E, Díaz I. 2007. Los costos clasificación y determinación económica agrícola. En: Economía Agrope- cuaria. La Habana, Cuba: Ed Félix Varela. p 58-64.

26. Valdés A, Sotolongo J, Escobar O. 2012. Incremento del peso corporal y prevención de los trastornos entéricos en crías porcinas en aplicar en cerdas gestadas contitucionales homeopáticas. V Seminario Internacional de Porcicultura Tropical. La Habana, Cuba.

27. Vidal F, Vasquez $R$, Leandro $R$, Pereira E, Peña I, del Toro A. 2005. Eficacia de la moxibustión y el Arsenicum album a la $30 \mathrm{CH}$ como terapia alternativa en el tratamiento del sindrome diarreico en cerdo. REDVET 6(2). [Internet] Disponible en: http:// www.veterinaria.org/revistas/redvet/ n020205/020509.pdf

28. Vidal F, Leandro R, Bazo Y. 2004. Evaluación del Arsenicum album como promotor del crecimiento en pesos en crías porcinas. REDVET 5(12). [Internet] Disponible en: http://www.veterinaria.org/ revistas/redvet/n121204.htm

29. Vidal F, Peña I, Vásquez R, González R, Pérez Y. 2006. Tratamiento del sindrome diarreico en crías porcinas utilizando medicameentos homeopáticos. REDVET 7(2). [Internet] Disponible en: http://www.veterinaria.org/revistas/ redvet/n020206/020605.pdf

30. Vidal F, Ruiz P, Ohilson L. 2014. Eficacia del Arsenicum album, phosphorus y Veratrum album en el tratamiento de la disenteria porcina en precebas porcinas. En: XXIV Congreso Panamericano de Ciencias Veterinarias. La Habana, Cuba.

31. Vidal F, Peña García I, de la Torres Canovas R. 2015. Efecto de medicamento homeopático empleado como promotores del incremento en peso en la crianza porcina de traspatio. En: VI Seminario Internacional de Porcicultura Tropical. La Habana, Cuba.

32. Vivanco J. 2002. El uso de la homeopatía en la avicultura. En: XVIII Congreso Panamericano de Ciencias Veterinarias. La Habana, Cuba. 